\title{
炔醇分子内环化促发的串联反应在螺杂环化合物合成中的应用
}

\author{
余述燕*,a

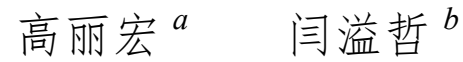 \\ 尹志刚 ${ }^{a}$ \\ 商永嘉 $*, c$

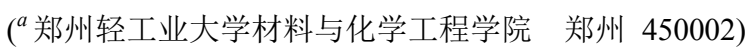 \\ ( ${ }^{b}$ 郑州轻工业大学食品与生物工程学院 郑州 450002)

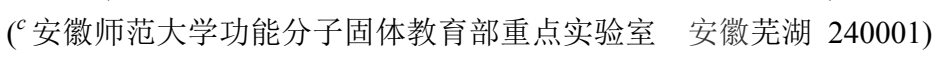

\begin{abstract}
摘要 螺杂环化合物独特的立体结构和丰富的理化特性, 激发了研究工作者对其高效合成方法的持续关注. 炔醇在过 渡金属作用下经 exo-dig 式分子内环化可原位形成环外烯醇梄, 其作为一类高活性的 $\mathrm{C} 2$ 合成子, 能够与同时具有亲电 和亲核特性的 “双亲性底物” 发生串联反应, 实现螺杂环骨架的快速构建. 综述了近年来炔醇分子内环化促发的串联 反应在螺杂环化合物合成中的应用进展，以期激发更多相关研究工作的设计与报道. 按照参与螺环构建的 “双亲性底 物”原子数目的不同进行分类, 重点阐述了反应采用的催化体系和反应机制, 分析了目前该领域存在的挑战, 并对未来 的发展进行了展望.
\end{abstract}

关键词＼cjkstart螺杂环；炔醇；串联反应; 环外烯醇梄

\section{Application of Cascade Reactions in the Synthesis of Sprio-hetero- cycles Initiated by Intramolecular Cyclization of Alkynols}

\author{
Yu, Shuyan ${ }^{*, a} \quad$ Gao, Lihong $^{a} \quad$ Yan, Yizhe $^{b} \quad$ Yin, Zhigang $^{a} \quad$ Shang, Yongjia ${ }^{*, c}$ \\ $\left({ }^{a}\right.$ College of Material and Chemical Engineering, Zhengzhou University of Light Industry, Zhengzhou 450002) \\ $\left({ }^{b}\right.$ College of Food and Bioengineering, Zhengzhou University of Light Industry, Zhengzhou 450002)
}

('Key Laboratory of Functional Molecular Solids, Ministry of Education, Anhui Laboratory of Molecule-Based Materials, College of Chemistry and Materials Science, Anhui Normal University, Wuhu, Anhui 241000)

\begin{abstract}
Due to their unique stereoscopic structure and rich physical and chemical properties, spiro heterocyclic compounds have aroused the continuous attention of researchers on their efficient synthesis methods. The exo-cyclic enol ethers generated in situ by exo-dig cyclization of alkynols under the promotion of transition metals could serve as $\mathrm{C} 2$ synthons to react with various "amphiphilic substrates" through cascade reactions mode. These cascade reactions could afford spiro heterocyclic skeletons in highly efficient and direct manners. In this paper, the application progress of cascade reactions in the synthesis of sprio-heterocycles initiated by intramolecular cyclization of alkynols is reviewed, which aims to stimulate the disclosure of more related research work. These work is elaborated according to the different atom numbers of the "amphiphilic substrates" involved in the construction of the spiro framworks. The catalytic system and reaction mechanism are mainly described, the challenges in this field are analyzed, and the future development is also put forward.
\end{abstract}

Keywords sprio-heterocycles; alkynols; cascade reactions; exo-cyclic enol ether

螺环化合物是指具有两个相互垂直的环平面且只 共用一个原子的特殊骨架化合物. 螺环化合物独特的三 维立体刚性结构和丰富的生理活性, 使其在高分子膨胀 剂、不对称催化、防火材料、电致发光材料、医药和农 药等方面都展现出了非常重要的用途 ${ }^{[1]}$. 其中含有氮、 氧或硫等原子的螺杂环化合物具有螺共轭、螺超共轭以
及异头效应等一般有机化合物不具备的特殊性质 ${ }^{[2]}$. 从 成药性角度分析，在杂环化合物中引入螺环骨架会产生 活性增强、药代动力学特性改善、化合物构型稳定增加 以及不易产生耐药性等优点，具备发展成为医药分子的 潜力 ${ }^{[3]}$. 因此螺杂环化合物在药物设计中的应用引起了 越来越多药物化学家的重视. 目前已有很多上市药物含

\footnotetext{
* Corresponding authors. E-mail: yushuyan_zzuli@163.com; shyj@mail.ahnu.edu.cn Received June 23, 2020; revised July 28, 2020; published online August 18, 2020.

Project supported by the National Natural Science Foundation of China (No. 21602207), the Doctoral Research Fund of Zhengzhou University of Light Industry (No. 2014BSJJ009) and the Young Backbone Teachers' Fund of Zhengzhou University of Light Industry (No. 2019XGGJS010).

国家自然科学基金(No. 21602207)、郑州轻工业大学博士科研基金(No. 2014BSJJ009)和郑州轻工业大学青年骨干教师(No. 2019XGGJS010)资助项目.
} 
有螺杂环骨架(图 1). 因此开发简便高效地合成螺杂环 化合物的方法具有非常重要的学术意义和实用价值.

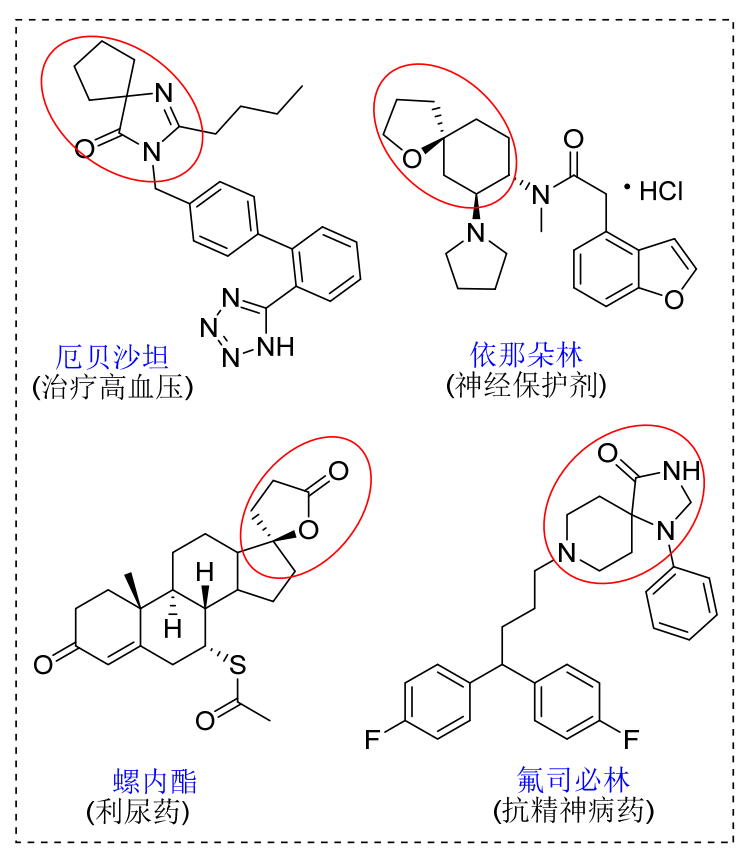

图式 1 一些具有螺杂环骨架的药物

Figure 1 Some drugs containing spiroheterocycle skeletons

已报道螺环化合物的主要合成方法，根据反应机制 的不同, 大概可以分为六类 (如 Scheme 2 所示 $)^{[4]}$ : (a)分 子间双取代反应; (b)金属促发的环化反应; (c)分子内取 代反应; (d)自由基环化反应; (e)分子内重排; (f)环加成 反应. 前五种反应模式 $(a \sim e)$ 大多需要预先制备复杂的 官能化底物, 且反应存在步骤繁多和收率不高等缺点. 而环加成反应(f)由于反应条件温和以及立体选择性好 等优点, 引起了越来越多研究工作者们的注意 ${ }^{[5]}$. 但是 由于环外双键底物通常不易获得，极大地限制了该方法 的广泛应用. 因此开发一种能够快速获得环外双键底物 并且能够容易实现结构多样性的方法, 将其应用于螺杂 环化合物的合成中, 具有十分重要的研究价值和应用潜 力.

\section{1 炔醇分子内环化促发的串联反应机制}

炔醇分子中碳碳参键在过渡金属活化下接受分子 内羟基进攻发生环化反应，依据区域选择性的不同，可 原位形成双键在环内和环外的两种环状烯醇醚，两者均 具有较高的反应活性，可与同时具有亲电和亲核性基团 的 “双亲性底物” 通过串联反应历程, 经氧鎓正离子中 间体转化为多取代含氧杂环化合物 ${ }^{[6]}$ (Scheme 3). 其中, 通过 exo-dig 环化模式产生的环外烯醇梄作为 $\mathrm{C} 2$ 合成子 与 “双亲性” 底物间的串联反应, 可一锅法高效地合成 具有螺环骨架的杂环化合物. 同时由于取代炔醇底物较

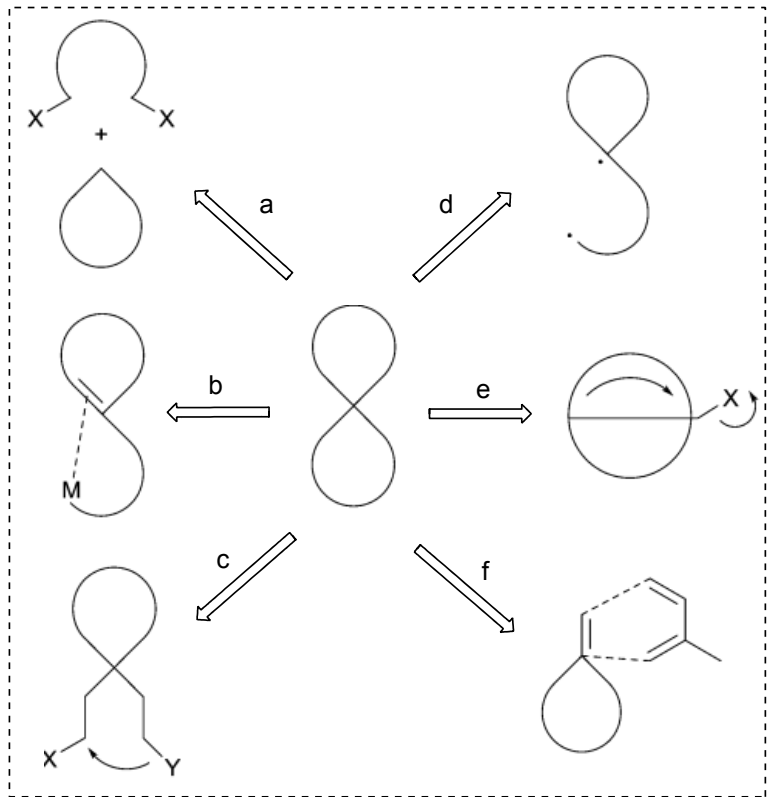

图式 2 螺环化合物的主要合成方法

Scheme 2 Synthetic methods for spirocycles

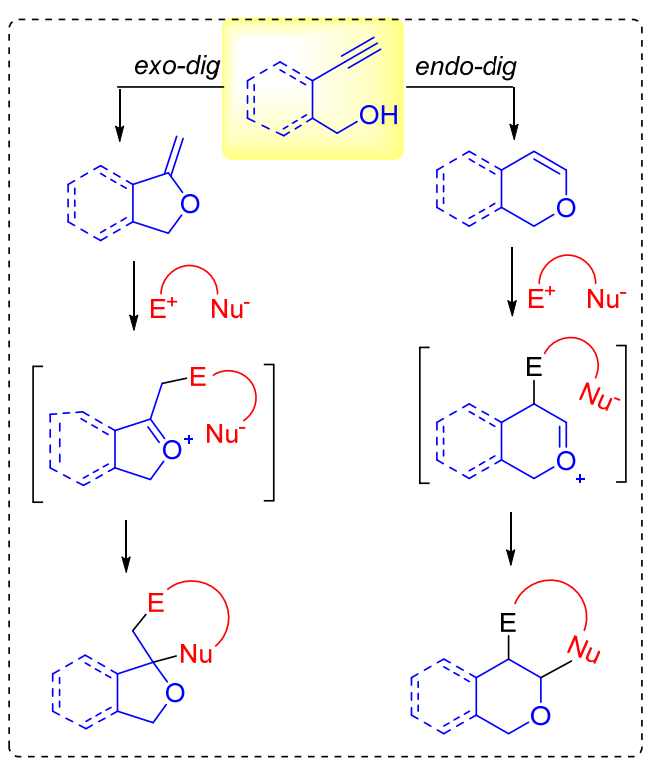

图式 3 炔醇分子内环化促发的串联反应原理

Scheme 3 Mechanism of cascade reactions involving intramolecular cyclization of alkynols

易获得，环化反应条件温和，该串联反应易于满足实现 产物结构多样性的要求，在分子库的建立和生物活性篮 选方面具有显著的优势. 本文按照参与反应的“双亲性” 底物的原子数目不同(包括 4 原子合成子和 3 原子合成 子), 综述了近年来炔醇分子内环化促发的串联反应在 螺杂环化合物合成中的应用进展，着重介绍了反应所采 用的催化体系，并对部分反应的机制进行了讨论，最后 结合本组在该领域的研究兴趣和经验，对该领域的发展 趋势进行了展望. 


\section{2 炔醇与 4 原子合成子的串联反应}

炔醇分子内环化原位形成的环外烯醇醚作为一类 特殊的富电子烯烃组分, 通过与一系列 4 原子合成砌块 或活性中间体的形式 $[4+2]$ 环化反应，在构建 $[5,6]$-螺杂 环化合物方面展现出令人满意的高效性和便利性.

喹啉类化合物通常具有丰富的药理活性, 因此对其 高效合成方法的研究一直是有机合成和药物开发领域 的关注热点 ${ }^{[7]}$. 其中芳亚胺与富电子烯烃的 Povarov 反 应是构建喹啉类化合物行之有效的策略之一 ${ }^{[8]} .2008$ 年, Fañanás 课题组 ${ }^{[9]}$ 发现炔醇、醛和胺在过渡金属与布朗 斯特酸协同催化作用下，通过原位形成环状烯醇醚与芳 亚胺的一锅法 Povarov 型反应, 高效地转化为呋喃螺喹 啉类化合物 (Scheme 4). 在反应机理方面, 作者提出了 串联反应历程: 首先原位形成的高活性铂催化剂活化炔 醇碳碳参键, 使其易于接受分子内羟基亲核进攻, 原位 生成环外烯醇醚中间体. 与此同时, 布朗斯特酸活化醛 基使其易于接受芳胺进攻, 原位生成芳亚胺中间体. 其 后通过两活性中间体间的 Mannich 型加成-环化串联过 程, 转化为呋喃螺喹啉化合物. 该方法的开发不仅为具 有类似核心骨架化合物的合成提供了便捷的路径, 而且 通过炔醇原位形成环状烯醇醚的策略突破了传统 Povarov 反应中官能化二氢呋喃难以获得的限制, 进一 步拓展了反应的底物范围, 满足了产物多样性的需求, 为构效关系的研究以及生物活性分子库的建立提供了 坚实的基础.

受上述工作启发, 2018 年, 柳凌艳课题组 ${ }^{[10]}$ 将高炔 丙胺在 $\mathrm{FeCl}_{3}$ 作用下原位形成的环亚胺正离子与炔醇在
$\mathrm{PtCl}_{2}$ 活化下原位形成富电子烯醇醚的策略相结合，同 样通过非经典 Povarov 反应构建了一系列螺吡咯喹啉类 化合物(Scheme 5).

螺缩酮不仅是许多生物活性天然产物的核心骨 架 ${ }^{[11]}$, 而且最近研究表明某些从天然产物衍生化的简单 螺缩酮仍能保留与天然产物类似的生物活性 ${ }^{[12]}$. 因此 已有很多关于类天然产物型螺缩酮衍生物作为口服先 导药物的报道, 其中 [5,6]-螺缩酮是螺缩酮家族中占比 最大, 也是研究最为广泛的一支. 传统合成 $[5,6]$-螺缩酮 化合物的方法是通过二羟基酮的分子内环化反应 ${ }^{[13]}$, 但需要通过较长的线性合成路线预先制备二羟基酮，这 给分子结构的修饰带来了极大的不便利性. 2009 年, Barluenga 课题组 ${ }^{[14]}$ 利用原位形成环外烯醇醚的串联反 应参键，通过水杨醛、芳胺与炔醇的三组分一锅法反应, 实现了 $[5,6]$ 螺缩酮类化合物的高效合成. 反应结束后虽 然首先得到的是两个非对映立体异构体混合物，但通过 一步简单的异构化处理, 可高收率地获得单一构型的螺 缩酮化合物(Scheme 6a). 此外, 芳胺组分还可替换为原 甲酸酯类化合物，在类似的反应条件下可以良好的收率 获得烷氧基取代的螺缩酮化合物(Scheme 6b). 与芳胺 参与的反应不同, 在作者探究的炔醇、水杨醛和原甲酸 酯的三组分反应中，只选择性地生成了单一构型产物， 这为底物控制的对映选择性合成提供了可能. 2013 年, 龚流柱课题组 ${ }^{[15]}$ 利用手性磷酸的空间诱导作用, 通过 与金的协同催化参键，实现了这类三组分反应的对映选 择性转化(Scheme 6c).

相比于金属与有机催化剂的共催化模式, 金属与金
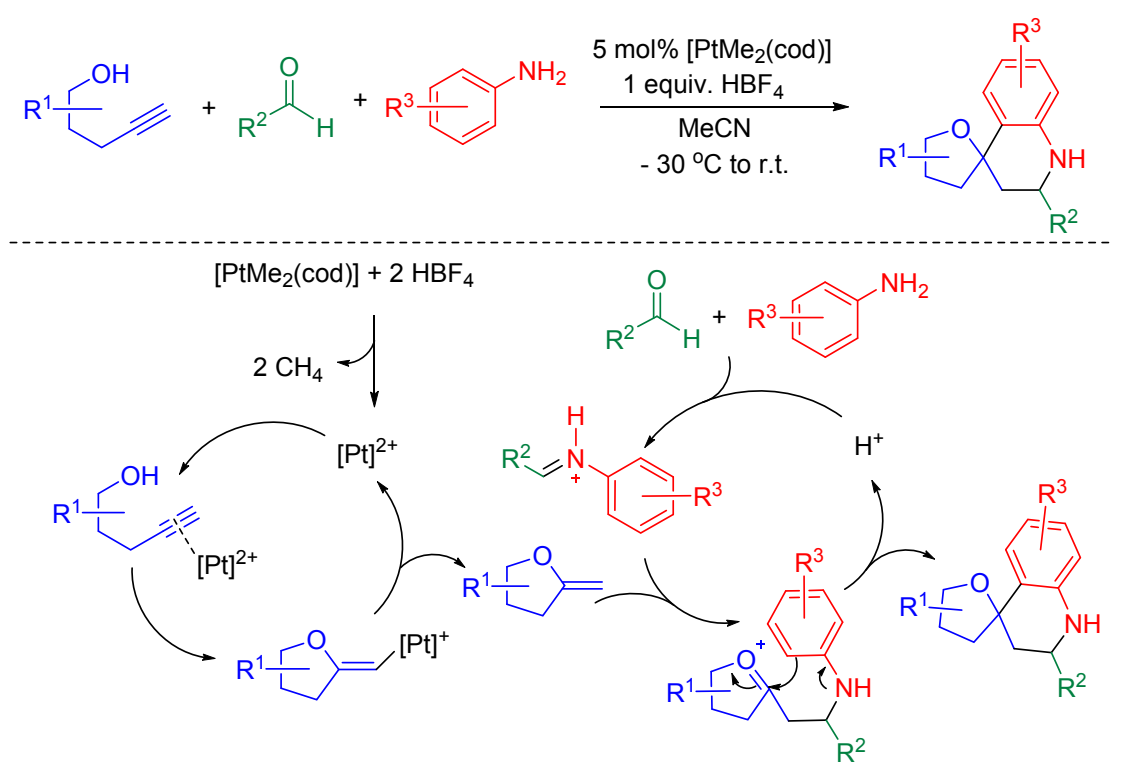

图式 4 炔醇、醛和芳胺的三组分反应

Scheme 4 Three-component reaction of alkynol, aldehyde and aromatic aniline 

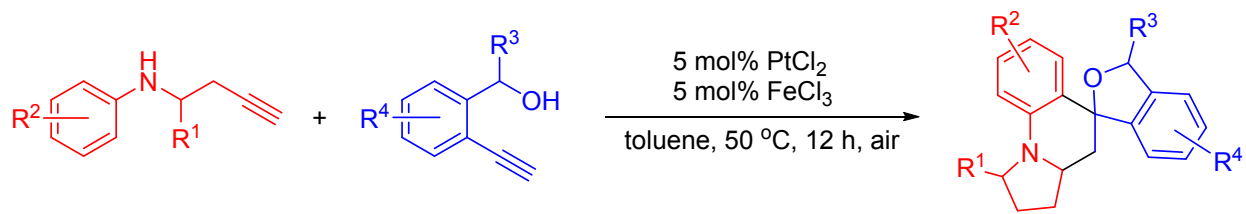

18 examples, $53 \% \sim 89 \%$ yield, $d r$ 3:1 15:1

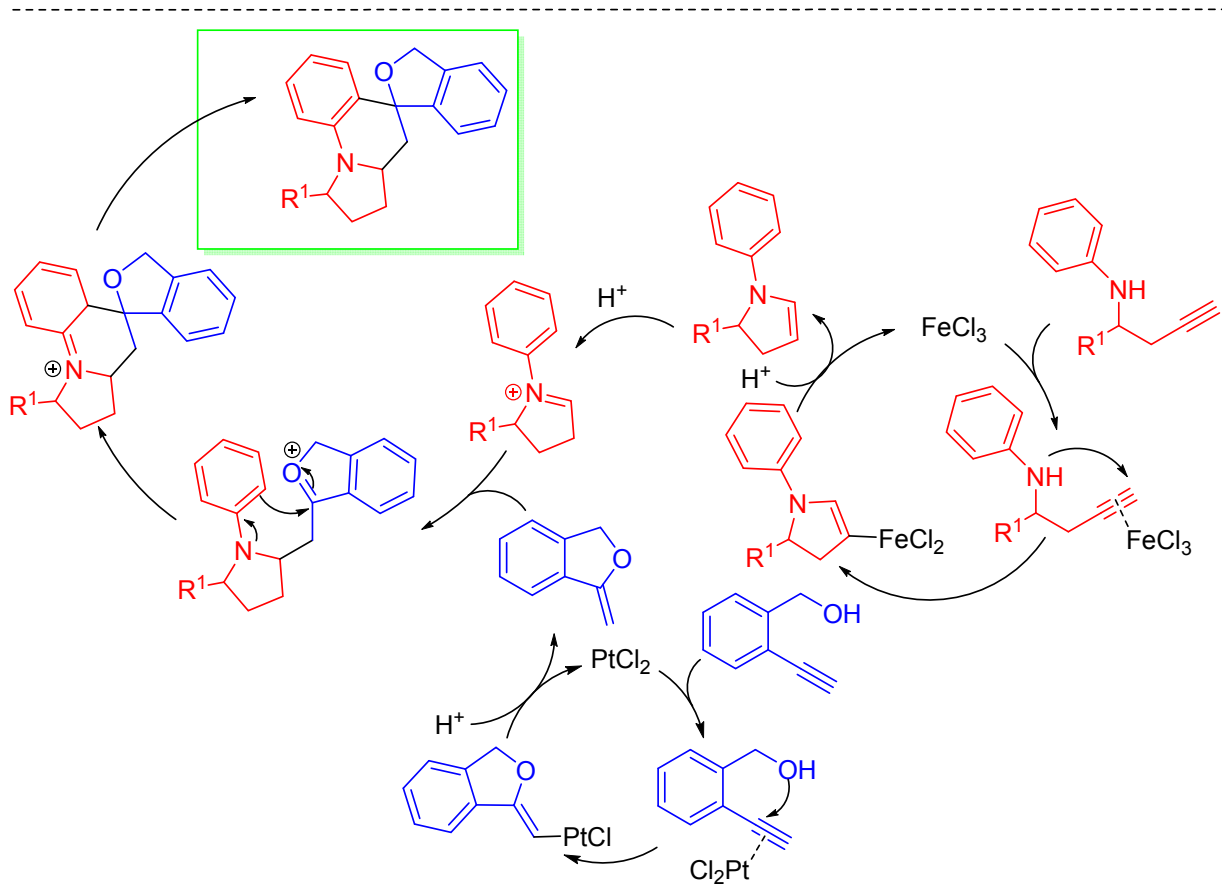

图式 5 炔醇和高炔丙胺的串联反应

Scheme 5 Cascade reaction of alkynol and homopropargylamine

属接力催化反应的研究相对较少, 可能是源于催化剂之 间的兼容性、催化剂活性匹配性以及反应选择性等问 题 ${ }^{[16]}$. 近年来, 徐政虎课题组在该领域做出了不少令人 关注的工作. 2014 年, 该课题组利用 $\mathrm{PPh}_{3} \mathrm{AuNTf}_{2}$ 与 $\mathrm{Y}(\mathrm{OTf})_{3}$ 的双金属接力催化模式, 实现了不饱和酮酸酯 与炔醇的环化反应，以 $81 \% \sim 91 \%$ 的收率合成了一系列 [5,6]-螺缩酮类化合物 (Scheme 7). 2016 年, 冯小明课题 组 ${ }^{[18]}$ 率先利用双金属金/手性 $N, N^{\prime}$-二氧化氮镍的串联催 化体系, 实现了炔醇与不饱和酮酯的不对称环化反应. 之后, 康强等 ${ }^{[19]}$ 利用金/手性铑催化剂的共催化模式, 同 样实现了 [5,6]-螺缩酮的不对称合成.

邻亚甲基苯醌 $(o-\mathrm{QM})$ 是一类重要的高活性合成中 间体. 独特的分子结构和多样的化学性质使其在众多领 域得到了广泛的应用 ${ }^{[20]}$. 受分子本身芳构化驱动力的 影响, 邻亚甲基苯醌可与亲核试剂发生迈克尔加成、与 亲双烯体发生环加成以及电环化等多种反应. 2017 年, 徐政虎课题组 ${ }^{[21]}$ 同样利用双金属共催化参键, 在 $\mathrm{PPh}_{3} \mathrm{AuCl}$ 和 $\mathrm{Sc}(\mathrm{OTf})_{3}$ 的催化下, 以邻羟基茮醇和炔醇 (或炔酰胺)为原料, 通过原位形成的邻亚甲基苯醌和环 外烯醇醚中间体的 [4+2]环化反应, 快速获得了一系列
[5,6]螺缩酮(或螺缩醛胺)化合物(Scheme 8).

此后不久, 石枫课题组 ${ }^{[22]}$ 更加系统地研究了多种 亚甲基苯醌与炔醇间的氧杂 $[4+2]$ 环化反应. 他们利用 均相 $\mathrm{Au}(\mathrm{I})$ 催化剂, 先后实现了对亚甲基苯醌衍生物、邻 高烯基亚甲基苯醌衍生物与邻羟基苄醇和炔醇的环化 反应，均以 $45 \% \sim 99 \%$ 的收率立体选择性地合成了一系 列具有不同取代基特性的 [5,6]螺缩酮化合物(Scheme 9).

2017 年, Gharpure 等 ${ }^{[23]}$ 也利用炔醇分子内环化促发 的串联反应合成了 $[5,6]$-螺缩酮化合物. 但是与上述所 有工作不同的是，该反应无需过渡金属的活化，仅在 TMSOTf 的催化下就完成了炔醇与水杨醛之间的氢烷 氧化-形式 $[4+2]$ 夰联反应，只是需要较长的反应时间. 此外，作者发现非末端炔醇也是合适的反应底物 (Scheme 10).

2006 年, Stierle 等 ${ }^{[24]}$ 从柏克利露天铜矿坑湖水中分 离得到了一种新的螺缩酮, 并将之命名为柏克利酸 (Berkelic Acid), 初步试验表明这种微生物来源的螺缩 酮对卵巢癌细胞株 OVCAR-3 的体外抑制浓度达到纳摩 尔级, 有望成为新一代的抗癌药物, 因此激发了众多研 究团队对其合成方法的研究. 


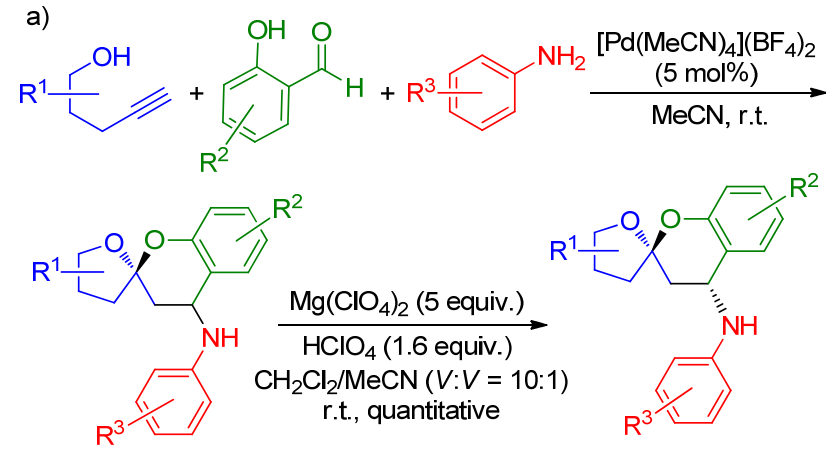

$71 \% \sim 90 \%$ yield, $d r=1: 1$

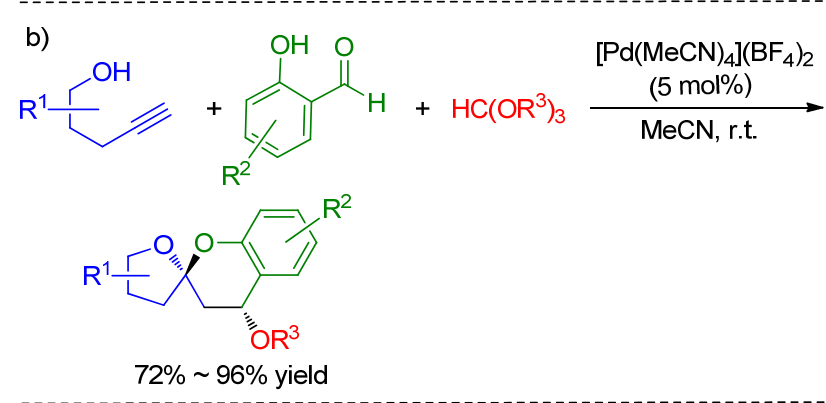

c)<smiles>[R][X]c1ccc(C#C)c(CO)c1</smiles>

$\mathrm{PPh}_{3}$ AuMe (5 mol\%), B*-H (10 mol\%) $15^{\circ} \mathrm{C}, 4$ A MS, 1,2,4-trichlorobenzene
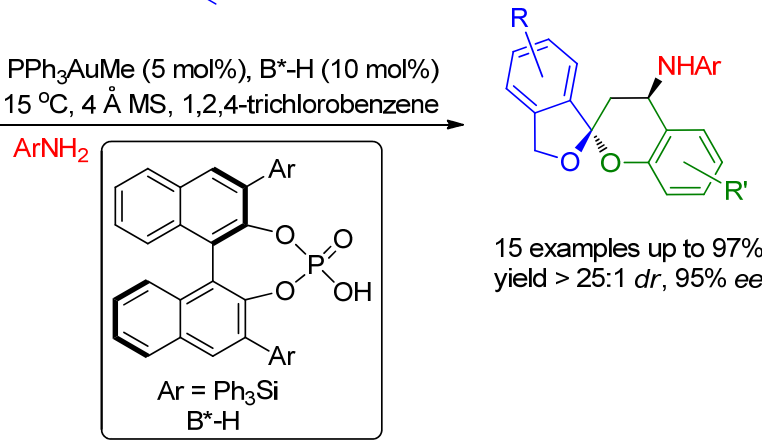

15 examples up to $97 \%$ yield $>25: 1 d r, 95 \%$ ee

图式 6 炔醇、水杨醛和芳胺(或原甲酸酯)的三组分反应

Scheme 6 Three-component reaction of alkynol, salicylaldehyde and aromatic aniline (ortho formate)

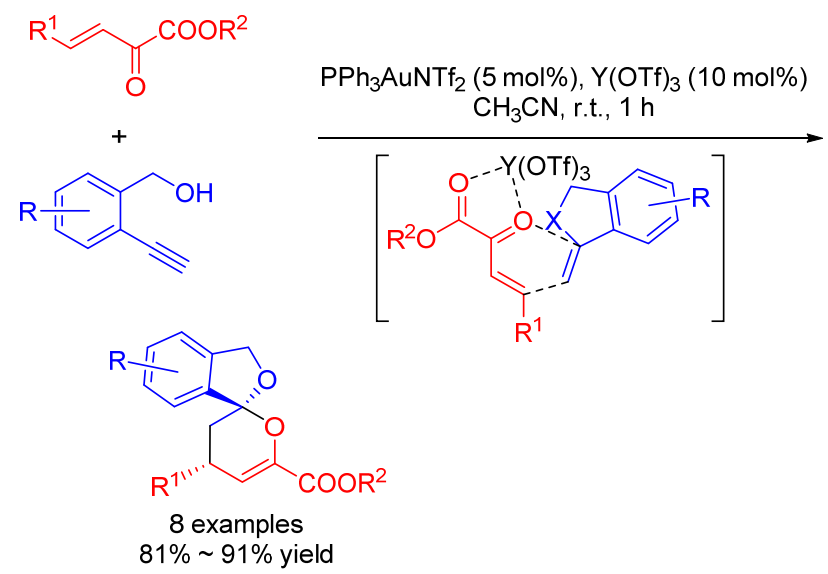

图式 7 炔醇与不饱和酮酸酯的串联反应

Scheme 7 Cascade reaction of alkynol and unsaturated ketoesters

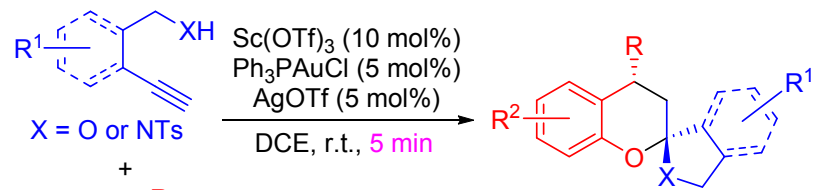

25 examples up to $97 \%$ yield<smiles>[R]C=C1C=CC(=O)C(CCCCCC)=C1</smiles>

图式 8 炔醇与邻羟基茮醇的串联反应

Scheme 8 Cascade reaction of alkynol and $o$-quinone methide

2016 年, Fañanás 研究团队 ${ }^{[25]}$ 报道了一步法合成柏 克利酸四环螺缩酮核心骨架的新方法，即以炔醇和邻炔 基水杨醛为底物，利用 AgOTf 的催化活化作用，通过原 位形成环外烯醇醚与异色满酮的形式[4+2]环化反应, 快速构建了柏克利酸类似物的分子库(Scheme 11).

嘧啶酮及其相关的杂环化合物大多具有重要的药 理特性，如抗癌、抗 HIV、抗菌和抗过敏等，因此在医 药和农药领域占有不可替代的重要地位 ${ }^{[26]}$. 迄今为止, 合成嘧啶酮最有效的方法莫过于醛、尿素(或硫脲)和可 烯醇化羰基化合物的三组分 Biginelli 反应 ${ }^{[27]}$. 但已报道 的羰基组分大多局限于酮类化合物或 1,3-二羰基化合 物，并且关于螺环嘧啶酮类化合物的合成还未见报道. 2020 年, 余述燕等将炔醇在过渡金属活化下原位形成 环外烯醇醚作为非传统的可烯醇化羰基组分，在过渡金 属氯化钯和三氟乙酸的共催化下, 实现了其与醛和尿素 (或硫脲)的三组分串联反应，以温和的条件， $45 \% \sim 87 \%$ 的收率和 $d r>20: 1$ 的立体选择性获得了一系列呋喃螺 嘧啶酮类化合物 ${ }^{[28]}$. 在相关控制实验的基础上，作者提 出了可能的反应机制: 首先醛和尿素(或硫艮)在三氟乙 酸的活化下生成酰基亚胺正离子中间体 $\mathbf{A}, \mathbf{A}$ 与原位形 成环外烯醇醚 B 的亲核加成反应生成中间体 $\mathbf{C}$, 氯化钯 诱导的双键异构化过程转变为环内烯醇醚中间体 $\mathbf{D}, \mathbf{D}$ 对活化芳醛亲核进攻生成氧鎓正离子 $\mathbf{E}$, 然后经分子内 亲核环化一质子化-脱水串联历程转变为目标产物呋喃 螺嘧啶酮类化合物(Scheme 12). 尝试了手性磷酸诱导 下的不对称反应，尽管尚未获得理想的对映选择性结 果，但有进一步优化的可能性.

\section{3 炔醇与 3 原子合成子的串联反应}

近年来, 环外烯醇醚作为一类高活性的 C2 合成子 与 3 原子合成砌块间的环化反应被逐渐地发掘出来, 在 合成具有呋喃骨架的五元螺环化合物方面发挥了重要 作用.

$[5,5]$ 螺缩酮广泛存在于天然产物和医药分子中，其 


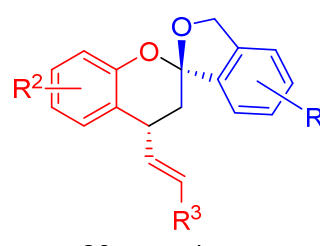

20 examples

$71-99 \%$ yield, up to $95: 5 d r$

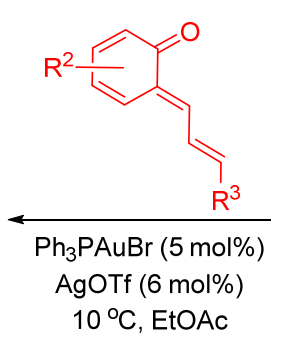

$10^{\circ} \mathrm{C}$, EtOAC

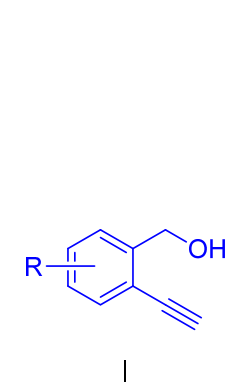

$\begin{array}{r}\text { AgOTf }(10 \mathrm{~mol} \%) \\ 10^{\circ} \mathrm{C}, \mathrm{CH}_{3} \mathrm{CN}\end{array} \mid$

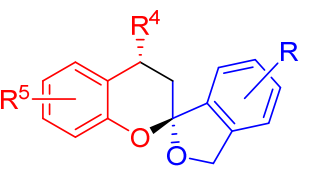

5 examples

$45 \% \sim 93 \%$ yield, up to $95: 5 d r$

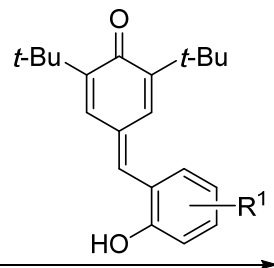

$\mathrm{Ph}_{3} \mathrm{PAuCl}(5 \mathrm{~mol} \%)$

AgOTf (6 mol\%)

$10^{\circ} \mathrm{C}$, EtOAC

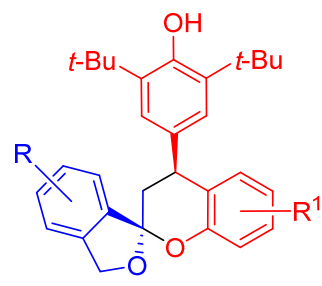

12 examples $80 \% \sim 96 \%$ yield, $>95: 5 d r$

图式 9 炔醇与多种亚甲基苯醌的串联反应

Scheme 9 Cascade reaction of alkynol and quinone methides

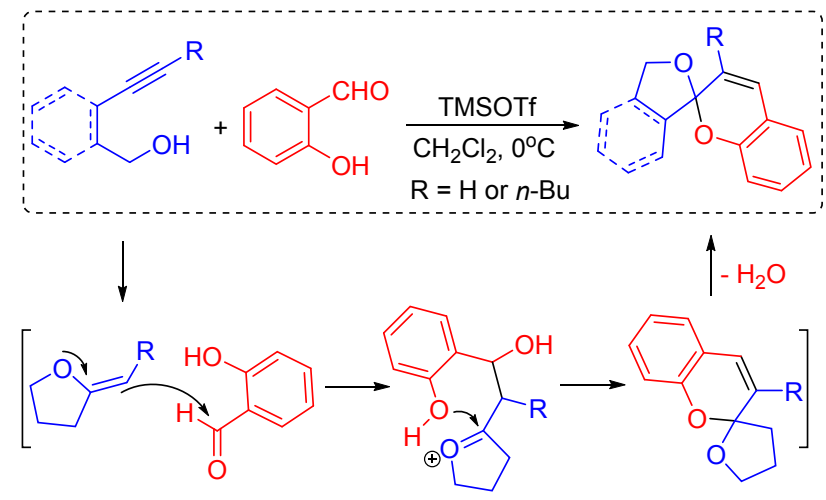

图式 10 炔醇与水杨醛的串联反应

Scheme 10 Cascade reaction of alkynol and salicylaldehyde

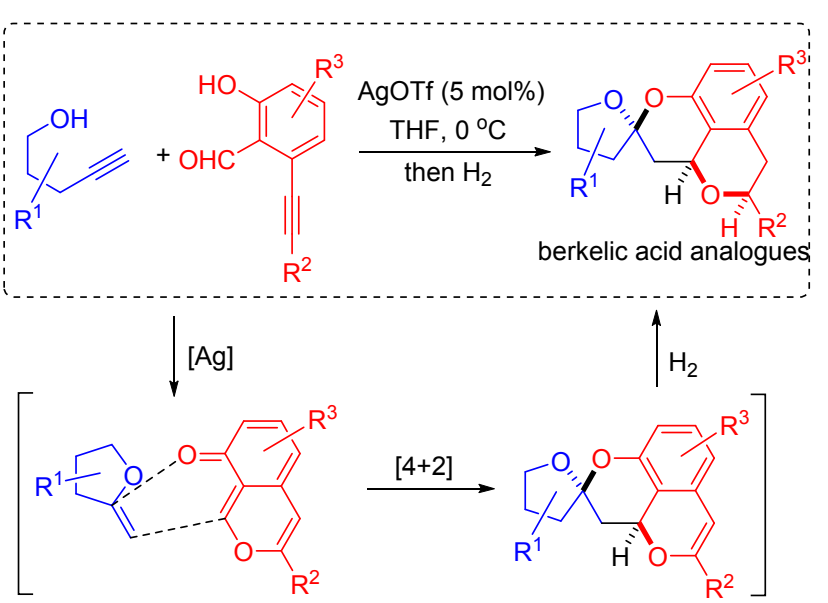

图式 11 炔醇和邻炔基水杨醛的串联反

Scheme 11 Cascade reaction of alkynol and ortho-alkynylsalicylaldehyde

重要而丰富的生理活性激发了众多合成工作者的研究

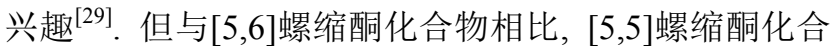
物的合成并不多见. 2013 年, Fañanás 课题组 ${ }^{[30]}$ 利用金催 化剂与手性磷酸共催化, 以炔醇、乙醛酸和胺为原料, 通过炔醇分子内环化原位形成的环外烯醇醚对羧基亚 胺中间体的亲核加成-环化串联反应，快速实现了 [5,5] 螺缩酮化合物的不对称合成(Scheme 13). 值得一提的 是, 合成的 $[5,5]$ 螺缩酮化合物含有 $\alpha$-氨基酸的结构, 为 产物的进一步衍生化提供了可能.

2017 年, Kontham 等 ${ }^{[31]}$ 以炔醇和酮酯为原料, $\mathrm{Bi}(\mathrm{OTf})_{3}$ 为催化剂, 发展了 [5,5]-螺缩酮化合物的新合成 方法(Scheme 14). 该反应具有广泛的底物普适性，作者 尝试了 42 种反应底物, 均以良好的收率获得了目标化 合物. 该反应的独特之处在于: $\mathrm{Bi}(\mathrm{OTf})_{3}$ 不仅作为过渡 金属催化剂, 活化碳碳参键, 促进炔醇分子内环化反应 生成环外烯醇醚，还作为路易斯酸催化剂，活化酮酯底 物中的羰基，使其易于接受烯醇醚的亲核进攻. 通过脱 水-水分子亲核加成-乙氧基消除串联历程，完成整个催 化循环.

徐政虎课题组 ${ }^{[32]}$ 长期从事炔醇分子内环化促进的 串联反应研究，并先后报道了环外烯醇醚这一高活性 C2 合成子与多种 C4 合成子间的环化反应，在 $[5,6]$ 螺缩 酮类化合物的合成方面做出了重要贡献. 受相关工作的 启发, 2018 年该课题组 ${ }^{[33]}$ 报道了一种合成 $[5,5]$ 螺缩酮化 合物的新方法，即通过炔醇与苯醌单亚胺的分子间环化 反应. 苯醌单亚胺是一类缺电子的高活性亲电试剂, 通 常作为 Diels-Alder 反应中的亲双烯体. 直到最近, 其作 为 3 原子合成子的潜力才被陆续地发掘出来 ${ }^{[34]}$. 在这项 新的工作中，徐政虎等 ${ }^{[33]}$ 用苯醌单亚胺去捕获环外烯 


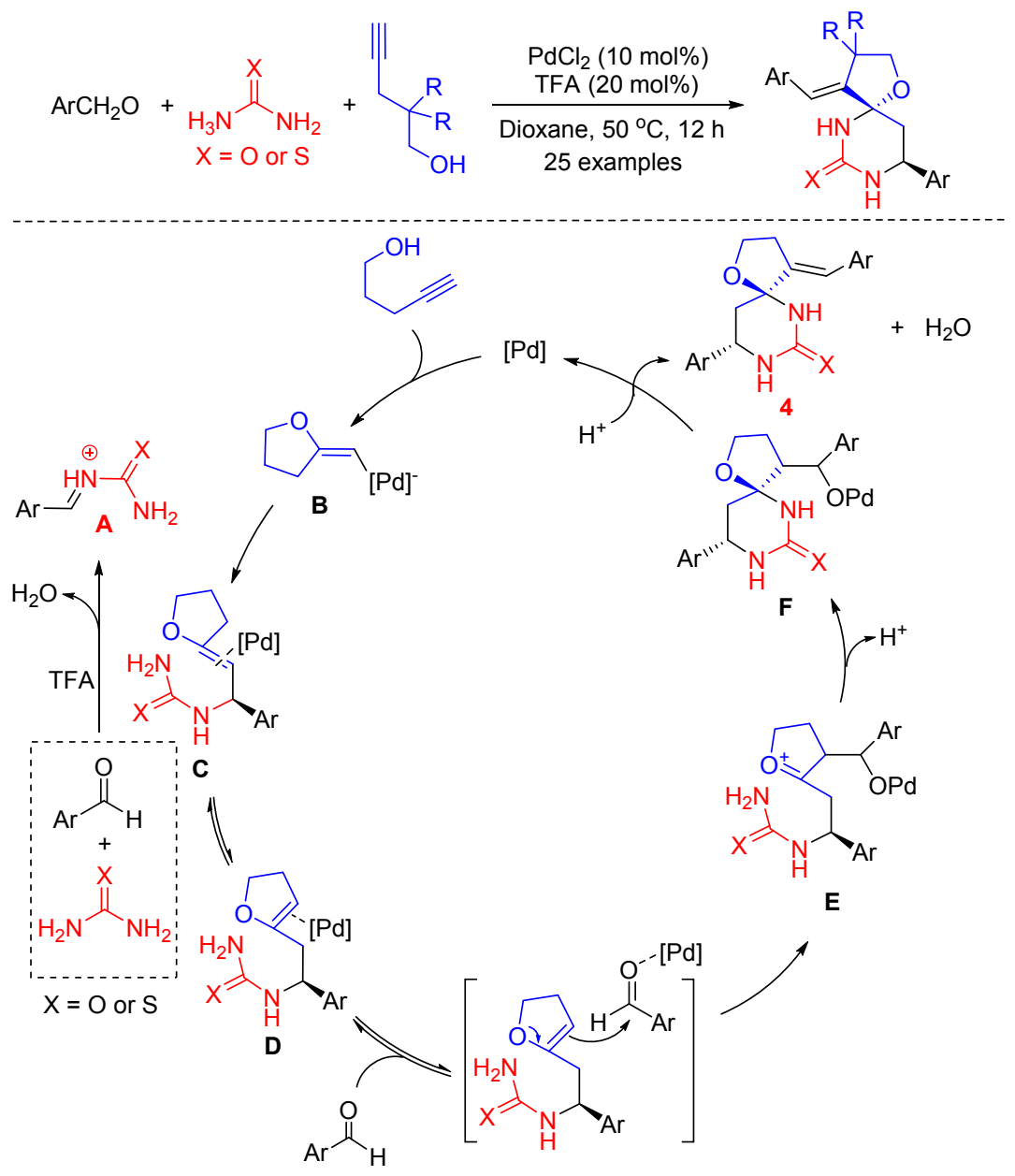

图式 12 炔醇、醛和尿素(硫艮)的三组分反应

Scheme 12 Three-component reaction of alkynol, aldehyde and urea (thiourea)

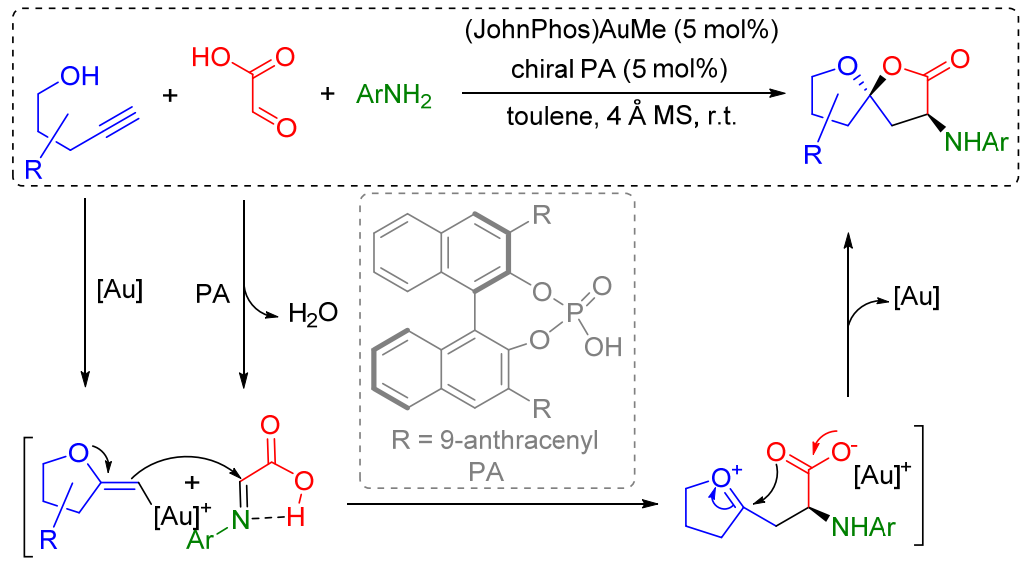

图式 13 炔醇、胺与乙醛酸的三组分反应

Scheme 13 Three-component reaction of alkynol, aniline and glyoxylic acid

醇醚, 通过加成环化串联反应历程, 简便地构建了一系 列[5,5]螺缩酮类化合物(Scheme 15).

五元氮杂螺环是众多具有生理活性天然产物、医药 和农药分子的重要结构基元 ${ }^{[35]}$. 目前常用的合成方法 大多涉及步骤繁多的反应转化, 因此发展简便高效合成
五元氮杂螺环化合物的新方法具有重要的研究价值和 应用前景. 氮杂环丙烷是有机合成领域一类非常实用的 合成砌块, 经常被用作 1,3-偶极子前体参与 $[3+2]$ 环加 成反应，构建五元含氮杂环化合物 ${ }^{[36]}$. 但由于 $\mathrm{C}-\mathrm{C}$ 键 裂解活化能较高 ${ }^{[37]}$, 目前大多反应是基于 $\mathrm{C}-\mathrm{N}$ 键裂解 

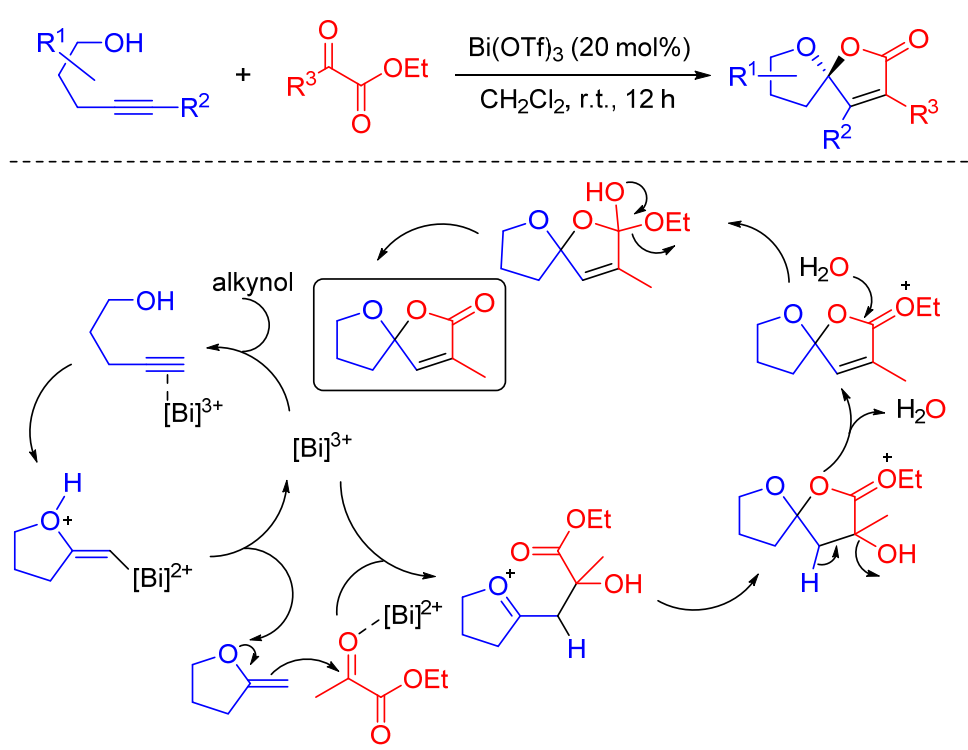

图式 14 炔醇与酮酯的串联反应

Scheme 14 Cascade reaction of alkynol and keto-ester

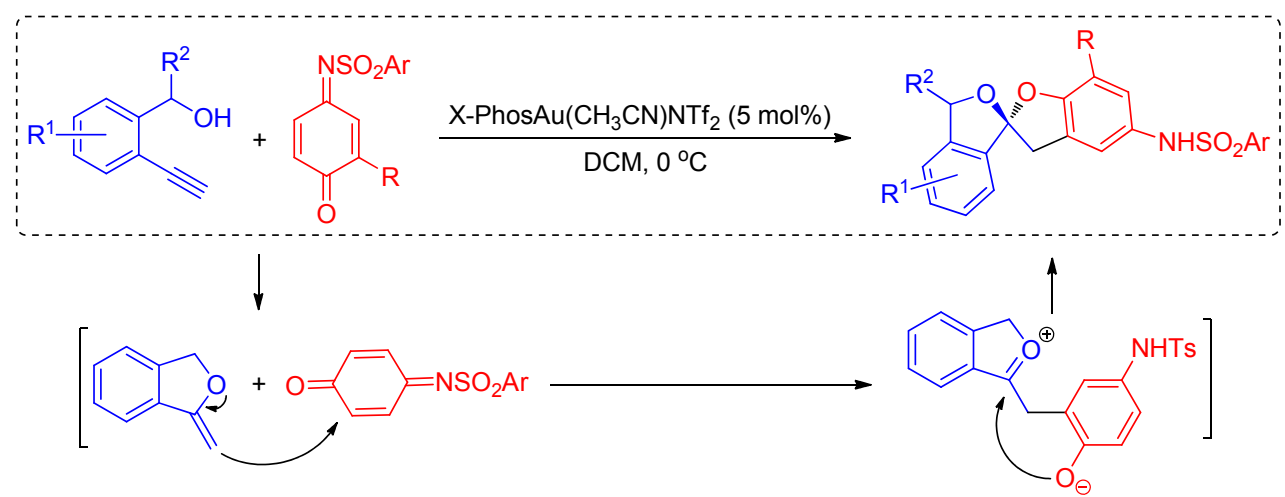

图式 15 炔醇与苯醌单亚胺的串联反应

Scheme 15 Cascade reaction of alkynols and quinone mo-noimines

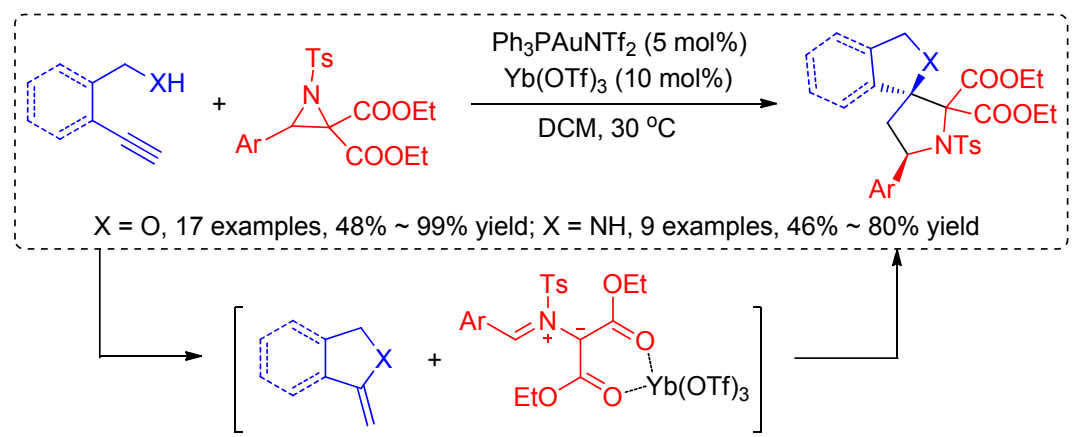

图式 16 炔醇与氮杂环丙烷的串联反应

Scheme 16 Cascade reaction of alkynols and aziridines

形成偶极子的环化反应 ${ }^{[38]} .2016$ 年, 徐政虎课题组 ${ }^{[39]}$ 以 炔醇和双酯基取代氮杂环丙烷为原料，通过金催化剂活 化炔醇碳碳参键形成环外烯醇醚中间体, 同时三氟甲磺 酸镱活化氮杂环丙烷, 选择性 $\mathrm{C}-\mathrm{C}$ 键裂解生成亚甲胺 叶立德中间体, 两活性中间体间的形式 $[3+2]$ 环加成反
应生成一系列五元氮杂螺环化合物(Scheme 16). 作者 认为氮杂环丙烷结构中两个酯基的存在, 有利于 $\mathrm{C}-\mathrm{C}$ 键的选择性裂解. 作者还尝试了添加手性噁唑啉配体来 调控反应的对映选择性，尽管只获得了 $39 \% e e$ ，但说明 了不对称转化的可行性以及进一步优化的可能性. 
2015 年, Krause 等 ${ }^{[40]}$ 以炔醇、醛(酮)和肼为原料, 通 过原位形成环外烯醇醚与亚胺叶立德的亲核加成一环化 反应, 合成了一系列具有吡唑结构的五元氮杂螺环化合 物(Scheme 17). 该反应条件温和, 底物普适性广, 产物 结构中的每个位置取代基均可通过底物的改变予以修 饰.

具有环戊烷结构的螺环骨架同样是很多天然产物 和生物活性分子的核心结构基元, 但由于长期以来缺乏 有效的合成策略，阻碍了其在众多方面的广泛应用 ${ }^{[41]}$. 2009 年, Iwasawa 等 ${ }^{[42]}$ 报道了铂(II)催化下丙二烯基硅醚 与烯醇醚间的 $[3+2]$ 环化反应. 在底物扩展时, 作者发 现炔醇也是合适的反应底物, 在 $\mathrm{PtCl}_{2}\left(\mathrm{C}_{2} \mathrm{H}_{4}\right)_{2}$ 作用下, 先转化为环状烯醇醚, 然后与丙二烯基硅醚反应生成呋 喃螺环戍烷衍生物(Scheme 18).

共轭烯炔酮分子结构中含有碳氧双键、碳碳双键、 碳碳参键, 是一类具有多反应位点的高活性反应底物. 自从 2004 年, Larock 等 ${ }^{[43]}$ 首次报道共轭烯炔酮在过渡金 属三氯化金作用下可发生分子内羰基氧对碳碳参键的 亲核环化反应，形成呋喃金碳正离子中间体，该中间体 可进一步与亲核试剂反应, 生成多取代呋喃衍生物. 该
反应具有条件温和、原子经济性高等优点，为构建具有 呋喃环骨架化合物提供了强有力的合成方法. 该工作一 经发现, 引起了众多研究工作者的关注, 并陆续报道了 许多有价值的研究成果 ${ }^{[44]}$. 2019 年, 徐政虎课题组 ${ }^{[45]}$ 将 共轭烯炔酮原位形成呋喃金碳正离子中间体与炔醇原 位形成环外烯醇醚的策略相结合，通过单一金属同时活 化两种底物的方式，实现了共轭烯炔酮与炔醇的分子间 环化反应，高效地构建了多取代环戊烷螺呋喃环化合物 (Scheme 19).

\section{4 挑战与展望}

综上所述，炔醇作为 $\mathrm{C} 2$ 合成砌块，与 4 原子或 3 原 子合成子间的形式 $[2+4]$ 和 $[2+3]$ 环化反应已经引起广 泛关注, 在相应螺杂环化合物的合成方面展现了重要的 应用价值. 从反应机制上看，4 原子合成子通常具有杂 双烯的结构特征，而部分 3 原子合成子具有 1,3-偶极子 的结构特征. 遗憾地是, 关于炔醇参与的其他 $[2+n]$ 环 化反应还未见报道. 除此之外, 本领域还面临以下几个 方面的挑战:

(1)目前已报道的工作集中在 $[5,6]$ 或 $[5,5]$-螺杂环的

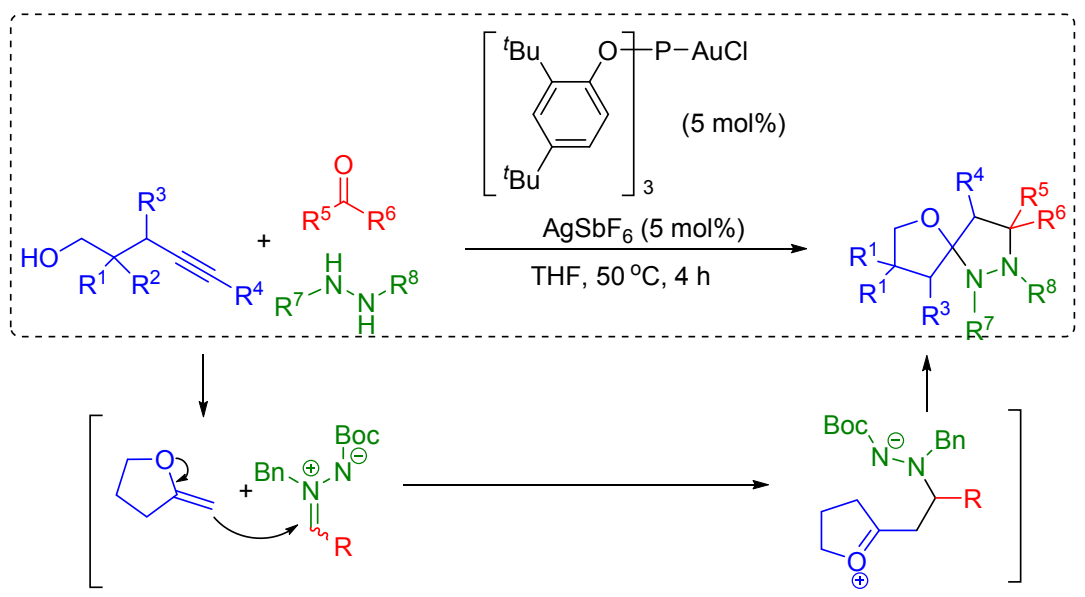

图式 17 炔醇、醛(酮)和肼的三组分反应

Scheme 17 Three-component reaction of alkynol, aldehyde (ketone) and hydrazine

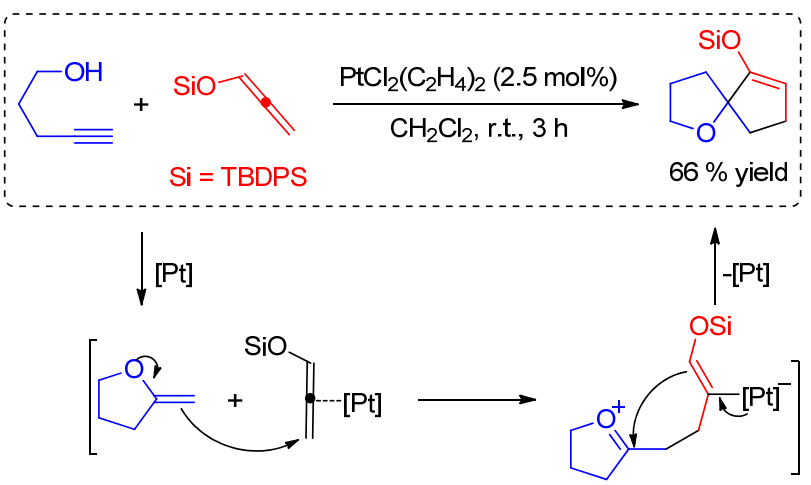

图式 18 炔醇与丙二烯基硅醚的串联反应

Scheme 18 Cascade reaction of alkynol and propadienyl silyl ethers 


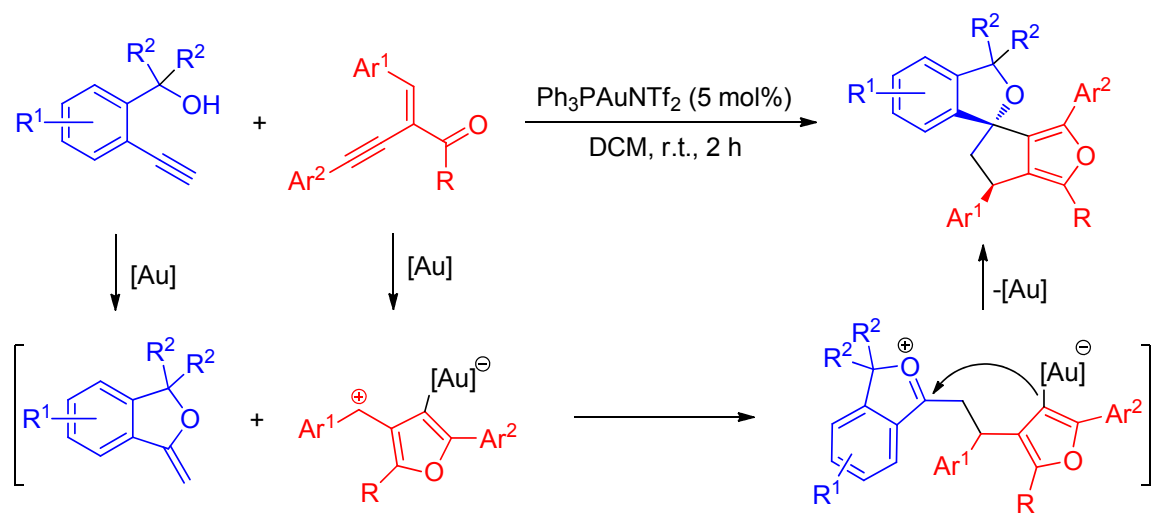

图式 19 炔醇与共轭烯炔酮的串联反应

Scheme 19 Cascade reaction of alkynols and alkynyl enones

合成中, 这主要是由于所报道反应中的炔醇组分基本上 局限于戊炔醇或其苯基类似物. 在过渡金属作用下, 通 过 exo-dig 环化模式只能形成五元环状烯醇醚, 进一步 的串联反应也只能构建 $[5, n]$-螺杂环化合物. 目前仅有 一例关于环已烯醇醚中间体的形成及其参与的串联反 应报道 ${ }^{[46]} .2009$ 年, Barluenga 等报道以烯炔醇为原料和 缺电子烯烃为原料, 在三氯化金的作用下经原位形成的 环状二烯中间体与亲双烯体的 $[4+2]$ 环化反应, 可快速 构建多环骨架化合物. 在底物扩展部分, 作者探究了一 例经历环外已烯醇醚的串联反应(Scheme 20).
(2)炔醇分子内区域选择性环化形成的环外烯醇醚, 在一定条件下会发生双键位置转移, 异构为环内烯醇 醚，导致螺环化合物难以形成或影响反应收率. 2008 年 Barluenga 课题组首次报道此串联反应参键时, 就发现 了这一现象. 在底物扩展时, 作者发现 4-戊炔-1-醇、苯 甲醛和苯胺的三组分反应并未按照预期的反应模式生 成呋喃螺氢化喹啉化合物, 而是意外地获得了呋喃并氢 化喹啉类衍生物. 作者 ${ }^{[9]}$ 认为并环化合物的生成是由原 位形成的环外烯醇醚在酸性条件下发生异构化导致的 (Scheme 21a).<smiles>C=C(C)C#CCCCCO</smiles>

图式 20 环外已烯醇醚中间体参与的反应

Scheme 20 Reaction involving cyclohexene alcohol ether intermediate<smiles>C#CCCCO</smiles>

a)

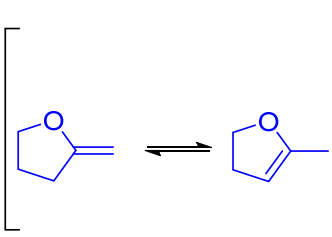

b)<smiles>C#Cc1ccccc1CO</smiles>

(No isomerization)

图式 21 4-戊炔-1-醇、苯甲醛和苯胺的三组分反应

Scheme 21 Three-component reaction of 4-pentyne-1-ol, benzaldehyde and aniline 
2013 年, 徐政虎课题组 ${ }^{[47}$ 还利用炔胺环化后生成 的环状烯胺的双键异构化反应的双键异构化反应，合成 了一系列并环缩醛胺化合物. 此后, $\mathrm{Xu}$ 课题组 ${ }^{[17]}$ 在探究 炔醇与不饱和酮酸酯的环化反应时, 为了避免原位形成 环外烯醇醚的双键移位问题，作者在炔醇底物中引入了 苯环结构, 保证了螺环骨架化合物的生成(Scheme 21b). 双键异构化的发生增加了反应的复杂性, 降低了反应收 率, 限制了反应底物的扩展. 因此在未来的研究中, 不 仅可以通过改变炔醇底物结构来限制烯醇醚中间体双 键的移位问题, 也可以通过反应条件的篮选(如催化剂 种类、反应介质、底物结构和活性等), 阐明双键异构化 发生的规律及影响因素, 为高效实现螺杂环化合物的设 计与合成提供指导.

(3)链状炔醇与依托于苯环的炔醇在反应性上的区 别尚不明晰, 为新反应的设计、条件优化以及底物扩展 等带来了不便. 在已报道的炔醇与 4 原子或 3 原子合成 子的 16 例串联反应中, 炔醇底物可分为两类: 链状炔醇 和依托于苯环的芳香炔醇, 其中 7 例反应只提及了链状 炔醇的反应情况 ${ }^{[14,25,28,30-31,39-40] ， 5}$ 例反应只拓展了芳基 炔醇类底物 ${ }^{[10,15,17,32,45]}$, 均未探究另一类炔醇的反应可 行性. 但已报道的反应实例和本课题组前期的研究结果 表明 ${ }^{[28]}$, 两类炔醇具有不同的反应特性, 遗憾的是目前 尚无文献对此进行系统地研究. 一般来说芳基炔醇具有 更高的反应活性 ${ }^{[10]}$, 且由于芳环空间位阻的影响, 芳基 炔醇参与的反应通常能够获得更加理想的立体选择 性 ${ }^{[9,40]}$.

\section{5 结论}

发展高效便捷的合成方法是保证螺杂环化合物丰 富应用价值得以实现的重要前提. 炔醇在过渡金属作用 下原位形成的环外烯醇醚与双亲性底物或中间体的串 联反应, 不仅能实现螺杂环骨架的快速构建, 还能最大 限度地满足产物结构的多样性要求, 这对活性物质的笁 选及其在相应领域的广泛应用均有重要的推动作用. 尽 管目前该领域仍存在不少尚未解决的挑战，但其显著的 优势一定会激发越来越多有意义工作的发掘与报道.

\section{References}

[1] (a) Krapcho, A. P. Synthesis 1974, 383.

(b) Lovering, F.; Bikker, J.; Humblet, C. J. Med. Chem. 2009, 52, 6752

(c) Nai, G. X.; Dai, R. Q. Technol. Dev. Chem. Ind. 2012, 41, 6 (in Chinese).

(赖谷仙，戴日强，化工技术与开发, 2012, 41, 6.)

(d) Zou, L.; Wang, C. S.; Liang, B. New Chem. Mater. 2013, 41, 12 (in Chinese).

(邹琳, 王长松, 梁兵, 化工新型材料, 2013, 41, 12.)

(e) Fan, K. Q.; Wang, X. B.; Ma, Y. P.; Yang, H. R.; Han, G. L.; Zhou, L. M.; Fang, S. M. New J. Chem. 2020, 44, 8351.
[2] (a) Wei, R. B.; Liang, Y. Chin. J. Org. Chem. 2008, 28, 1501 (in Chinese).

(魏荣宝, 梁娅, 有机化学, 2008, 28, 1501.)

(b) Li, F. F.; Wang, L.; Chen, S. Y.; Zhu, H. M.; Ouyang, G. P. Chem. Res. Appl. 2012, 24, 1181 (in Chinese).

(李飞飞, 王磊，陈舒忆，朱红梅，欧阳贵平，化学研究与应用， 2012, 24, 1181.)

(c) Wei, R. B.; Zhang, D. W.; Liu, B.; Liu, Y.; Jia, C. X. Chin. J. Org. Chem. 2009, 29, 517 (in Chinese)

(魏荣宝, 张大为, 刘博, 刘洋, 李文丽, 贾辰熙, 有机化学, 2009, 29, 517.)

(d) Michael, J. B. Carbon 1997, 35, 1.

[3] Rios, R. Chem. Soc. Rev. 2012, 41, 1060.

[4] Kotha, S.; Panguluri, N. R.; Ali, R. Eur. J. Org. Chem. 2017, 5316.

[5] Li, X. F. Ph.D. Dissertation, Tianjing University, Tianjing, 2003 (in Chinese).

(李澓芳, 博士论文, 天津大学, 天津, 2003.)

[6] (a) Alcaide, B.; Almendros, P.; Alonso, J. M. Molecules 2011, 16, 7815 .

(b) Zeng, X. M. Chem. Rev. 2013, 113, 6864.

(c) Alcaide, B.; Almendros, P. Acc. Chem. Res. 2014, 47, 939.

[7] (a) Solomon, V. R.; Lee, H. Curr. Med. Chem. 2011, 18, 1488

(b) Afzal, O.; Kumar, S.; Haider, M. R.; Ali, M. R.; Kumar, R.; Jaggi, M.; Bawa, S. Eur. J. Med. Chem. 2015, 97, 871.

[8] (a) Kouznetsov, V. V.; Mendez, L. Y. V.; Gomez, C. M. M. Curr. Org. Chem. 2005, 9, 141.

(b) Kouznetsov, V. V. Tetrahedron 2009, 65, 2721.

(c) Fochi, M.; Caruana, L.; Bernardi, L. Synthesis 2014, 46, 135.

[9] Barluenga, J.; Mendoza, A.; Rodríguez, F.; Fañanás, F. J. Angew. Chem. Int. Ed. 2008, 47, 7044.

[10] Wang, L.; Liu, L. Y.; Chang, W. X.; Li, J. J. Org. Chem. 2018, 83, 7799.

[11] Aho, J. E.; Pihko, P. M.; Rissa, T. K. Chem. Rev. 2005, 105, 4406.

[12] (a) Uckun, F. M.; Mao. C.; Vassilev, A. O.; Huang, H.; Jan, S. T. Bioorg. Med. Chem. Lett. 2000, 10, 541.

(b) Barun, O.; Kumar, K.; Sommer, S.; Langerak, A.; Mayer, T. U.; Muller, O.; Waldman, H. Eur. J. Org. Chem. 2005, 4773.

[13] Mead, K. T.; Brewer, B. N. Curr. Org. Chem. 2003, 7, 227.

[14] Barluenga, J.; Mendoza, A.; Rodríguez, F.; Fañanás, F. J. Angew. Chem. Int. Ed. 2009, 48, 1644.

[15] Wu, H.; He, Y. P.; Gong, L. Z. Org. Lett. 2013, 15, 460.

[16] Liang, M. M.S. Thesis, Shangdong University, Jinan, 2018 (in Chinese).

(梁曼, 硕士论文, 山东大学, 济南, 2018.)

[17] Wang, X. H.; Dong, S. L.; Yao, Z. L.; Feng, L.; Daka, P.; Wang, H.; $\mathrm{Xu}, \mathrm{Z}$. H. Org. Lett. 2014, 16, 22

[18] Li, J.; Lin, L.; Hu, B.; Lian, X.; Wang, G.; Liu, X; Feng, X. Angew. Chem. Int. Ed. 2016, 55, 6075

[19] Gong, J.; Wan, Q.; Kang, Q. Adv. Synth. Catal. 2018, 360, 4031.

[20] (a) Willis, N. J.; Bray, C. D. Chem.-Eur. J. 2012, 18, 9160. (b) Bai, W. J.; David, J. G.; Feng, Z. G.; Weaver, M. G. Wu, K. L.; Pettus, T. R. R. Acc. Chem. Res. 2014, 47, 3655.

(c) Caruana, L.; Fochi, M.; Bernardi, L. Molecules 2015, 20, 11733; d) Ai, W.; Liao, D.; Lei, X. Chin. J. Org. Chem. 2015, 35, 1615 (in Chinese). (艾文英，廖道红，雷晓光，有机化学, 2015, 35, 1615.)

[21] Liang, M.; Zhang, S.; Jia, J.; Tung, C. H.; Wang, J. W.; Xu, Z. H. Org. Lett. 2017, 19, 2526.

[22] Wang, C. S.; Cheng, Y. C.; Zhou, J.; Mei, G. J.; Wang, S. L.; Shi, F. J. Org. Chem. 2018, 83, 13861.

[23] Gharpure, S. J.; Nanda, S. K.; Shelke, Y. G. Chem.-Eur. J. 2017, 23 , 10007.

[24] Stierle, A. A.; Stierle, D. B.; Kelly, K. J. Org. Chem. 2006, 71, 5357.

[25] Arto, T.; Santa-Marĭa, I. S.; Chiara, M. D.; Fañanás, F. J.; Rodríguez, F. Eur. J. Org. Chem. 2016, 5876.

[26] (a) Elsharif, A. M. Orient. J. Chem. 2019, 35, 658. (b) de Fatima, A.; Braga, T. C.; Neto, L. D. S.; Terra, B. S.; 
Oliveira, B. G. F.; da Silva, D. L.; Modolo, L. V. J. Adv. Res. 2015, 6,363 .

(c) Wang, B. Z.; Xu, X. Y.; Tao, Y. C.; Ke, S. Y.; Qian, X. H.; Li, Z. Chin. J. Pestic. Sci. 2010, 12, 429 (in Chinese).

(王宝珠, 徐晓勇, 陶玉成, 柯少勇, 钱旭红, 李忠, 农药学学报, 2010, 12, 429.)

[27] (a) Kappe, C. O. Acc. Chem. Res. 2000, 33, 879.

(b) Gong, L. Z.; Chen, X. H.; Xu, X. Y. Chem.-Eur. J. 2007, 13, 8920 .

(c) Heravi, M. M.; Moradi, R.; Mohammadkhani, L.; Moradi, B. Mol. Diversity 2018, 22, 751.

[28] (a) Yu, S. Y.; Wu, J. X.; Lan, H. B.; Gao, L. H.; Qian, H. Y.; Fan, K. Q.; Yin, Z. G. Org. Lett. 2020, 22, 102.

(b) Yu, S. Y.; Gao, L. H.; Lan, H. B.; Qian, H. Y.; Yin, Z. G.; Shang, Y. J. Chin. J. Org. Chem. 2020, 40, 2714 (in Chinese).

(余述燕, 高丽宏, 兰宏兵, 钱恒玉, 尹志刚, 商永嘉, 有机化学, 2020, 40, 2714.)

(c) Yu, S. Y.; Wu, J. X.; Lan, H. B.; Xu, H. W.; Shi, X. F.; Zhu, X. W.; Yin, Z. G. RSC Adv. 2018, 8, 33968.

(d) Yu, S. Y.; Gao, L. H.; Wu, J. X.; Lan, H. B.; Ma, Y.; Yin, Z. G. Chem. Pap. 2020, 74, 3303.

(e) Yu, S. Y.; Wu, J. X.; He, X. W.; Shang, Y. J. Appl. Organomet. Chem. 2018, 32, e4156.

(f) Yu, S. Y.; Zhang, Z. Q.; Yu, Z. Y.; Shang, Y. J. Appl. Organomet. Chem. 2014, 28, 657.

[29] (a) Yao, H. L.; Wang, J.; Tong, R. B. Chem. Rev. 2017, 17, 1109. (b) Wang, J.; Tong, R. B. J. Org. Chem. 2016, 81, 4325.

[30] Cala, L.; Mendoza, A.; Fañanás, F. J.; Rodríguez, F. Chem. Commun. 2013, 49, 2715.

[31] Kambale, D. A.; Thorat, S. S.; Pratapure, M. S.; Gonnade, R. G.; Kontham, R. Chem. Commun. 2017, 53, 6641.

[32] Teng, Q.; Qi, J. L.; Zhou, L.; Xu, Z. H.; Tung, C. H. Org. Chem. Front. 2018, 5, 990.

[33] (a) Jackson, S. K.; Banfield, S. C, Kerr, M. A. Org. Lett. 2005, 7, 1215 .

(b) Uliana, M. P.; Servilha, B. M.; Alexopoulos, O.; de Oliveira, K. T.; Tormena, C. F.; Ferreira, M. A. B.; Brocksom, T. J. Tetrahedron 2014, 70, 6963.

[34] (a) Liao, L. H.; Shu, C.; Zhang, M. M.; Liao, Y. J.; Hu, X. Y.; Zhang, Y. H.; Wu, Z. J.; Yuan, W. C.; Zhang, X. M. Angew. Chem. Int. Ed. 2014, 53, 10471.

(b) Sun. X. X.; Zhang, H. H.; Li, G. H.; Meng, L.; Shi, F. Chem. Commun. 2016, 52, 2968. (c) Shu, C.; Liao, L. H; Liao. Y. J.; Hu, X. Y.; Zhang, Y. H.; Yuan, W. C.; Zhang, X. M. Eur. J. Org. Chem. 2014, 4467.

[35] (a) Jasper, A.; Schepmann, D.; Lehmkuhl, K.; Vela, J. M.; Buschmann, H.; Holenz, J.; Wünsch, B. Eur. J. Med. Chem. 2012, 53, 327.

(b) Strachan, J.; Farias, J. J.; Zhang, J.; Caldwell, W. S.; Bhatti, B. S. Bioorg. Med. Chem. Lett. 2012, 22, 5089.

[36] Sweeney, J. B. Chem. Soc. Rev. 2002, 31, 247.

[37] Huisgen, R.; Scheer, W.; Mäder, H. Angew. Chem. Int. Ed. 1969, 8, 602.

[38] (a) Lu, P. F. Tetrahedron 2010, 66, 2549.

(b) Pineschi, M. Eur. J. Org. Chem. 2006, 4979

(c) Schneider, C. Angew. Chem. Int. Ed. 2009, 48, 2082

[39] Wang, B.; Liang, M.; Tang, J.; Deng, Y. T.; Zhao, J. H.; Sun, H.; Tung, C. H.; Jia, J.; Xu, Z. H. Org. Lett. 2016, 18, 4614.

[40] Wagner, B.; Hiller, W.; Ohno, H.; Krause, N. Org. Biomol. Chem. 2016, 14,1579

[41] (a) Sun, W. S.; Zhu, G. M.; Wu, C. Y.; Hong, L.; Wang, R. Chem.Eur. J. 2012, 18, 6737 .

(b) Singh, K.; Pramanik, S.; Hamlin, T. A.; Mondal, B.; Das, D.; Saha, J. Chem. Commun. 2019, 55, 7069.

[42] Kusama, H.; Ebisawa, M.; Funama, H.; Iwasawa, N. J. Am. Chem. Soc. 2009, 131, 45, 16352.

[43] Yao, T.; Zhang, X.; Larock, R. C. J. Am. Chem. Soc. 2004, 126, 40, 11164.

[44] (a) Patil, N. T.; Wu, H.; Yamamoto, Y. J. Org. Chem. 2005, 70, 4531.

(b) Oh, C. H.; Reddy, V. R.; Kim, A.; Rhim, C. Y. Tetrahedron Lett. 2006, $47,5307$.

(c) Liu, Y. H.; Zhou, S. Org. Lett. 2005, 6, 4609.

(c) Liu, F. Ph.D. Dissertation, East China Normal University, Shanghai, 2013 (in Chinese).

(刘锋, 博士论文, 华东师范大学, 上海, 2013.)

(d) Di, X. Y. Ph. D. Dissertation, East China Normal University, Shanghai, 2019 (in Chinese).

(底晓显，博士论文，华东师范大学，上海, 2019.)

[45] Qi, J. L.; Teng, Q.; Thirupathi, N.; Tung, C. H.; Xu, Z. H. Org. Lett. 2019, 21, 692.

[46] Barluenga, J.; Calleja, J.; Mendoza, A.; Rodrígue, F.; Fañanás, F J. Chem.-Eur. J. 2010, 16, 7110.

[47] Wang, X.; Yao, Z.; Dong, S.; Wei, F.; Wang, H.; Xu, Z. H. Org. Lett. 2013, 15, 2234. 